\title{
Educación para la protección de los datos personales de los niños y adolescentes en la red
}

\author{
Asociación Asecum ${ }^{1}$, Superintendencia de Industria y Comercio ${ }^{2}$ \\ ${ }^{1}$ Cra. 69 No. 96-17- La Alborada-Cundinamarca-Bogotá-Colombia \\ beimar.castro@unimilitar.edu.co, claudia.lineros@unimilitar.edu.co
}

\begin{abstract}
The article argues the process of creating the virtual course: Education for the Protection of Personal Data of Children and Adolescents on the Internet, designed by the Superintendency of Industry and Commerce of Colombia, in order to contribute to the training of parents, tutors or teachers responsible handling of personal data of children and adolescents of school age and the prevention of crimes related to the violation of fundamental rights.
\end{abstract}

Resumen. El artículo evidencia el proceso de creación del curso virtual: Educación para la protección de los datos personales de los niños y adolescentes en la Red, diseñado por la Superintendencia de Industria y Comercio de Colombia, con el propósito de contribuir a la formación de padres, tutores o docentes en el manejo responsable de los datos personales de los niños y adolescentes en edad escolar y en la prevención de delitos relacionados con la violación de los derechos fundamentales.

\section{Escenario de uso}

Es política del estado colombiano, dentro de su Plan Nacional de Desarrollo: Todos por un nuevo país: Paz, Equidad y Educación, promover una cultura de la convivencia pacífica y de la tolerancia. Después de casi cincuenta años de conflicto armado en Colombia, la sociedad y el Estado le han apostado a la proyección de un futuro pacífico, a través de la consolidación de una sociedad más capacitada y educada para enfrentar los retos del posconflicto y del siglo XXI. Como uno de los ejes centrales de este propósito se ha erigido la educación en todas sus manifestaciones: primaria y básica, media, superior, y la educación para el trabajo y el desarrollo humano.

Es así como a la tarea de la educación se le han sumado algunas agencias del Estado que cumplen con otros propósitos, pero que contribuyen a incentivar la protección de todos sus ciudadanos y, con mayor énfasis, la de los niños y adolescentes. Una de dichas agencias es la Superintendencia de Industria y Comercio (SIC), la cual tiene como objetivo fundamental salvaguardar los derechos de los consumidores, proteger la libre y sana competencia, actuar como autoridad nacional de la propiedad industrial y defender los derechos fundamentales relacionados con la correcta administración de datos personales.

A propósito de este último tema, es de conocimiento generalizado que las redes sociales se han convertido en un medio importante de socialización y divulgación del conocimiento, pero también en una amenaza para niños y adolescentes quienes, desconocedores de los riesgos latentes en Internet, en ocasiones, comparten sus datos e información personal, poniendo en riesgo su identidad, su imagen y, en algunos casos, su salud y bienestar. Por tal razón, la SIC, a través de su programa SICEDUCA, ha venido 


\section{CBIE-LACLO 2015}

Anais dos Workshops do IV Congresso Brasileiro de Informática na Educação (CBIE 2015)

organizando cursos y seminarios presenciales a lo largo y ancho del país, en los que ha capacitado a miles de personas, incluso niños y adolescentes.

Es en este contexto que nace la idea de un MOOC que ofrezca herramientas a padres, tutores o docentes de niños y adolescentes en edad escolar para el manejo adecuado de sus datos personales. Este curso consta de un módulo introductorio y cinco temáticos; los temas que se tratan son: intimidad y protección de datos; interacción y riesgos en Internet; y pautas para padres y docentes.

El curso es diseñado por ingenieros y pedagogos de la SIC especializados en el tema y se soporta en el nuevo régimen legal establecido por la Ley 1581 de 2012 y los nuevos planteamientos y retos que se establecen para el manejo adecuado de los datos personales en Colombia.

Pedagógicamente, el curso aprovecha recursos de la virtualidad como videos infográficos, llamados de alerta, hipervínculos, glosarios, actividades de autoformación y juegos lúdicos, todos ellos para gestionar y promover el aprendizaje autónomo. Además, se ofrece al usuario un mapa conceptual que condensa los conceptos más importantes de cada módulo. Esta herramienta facilita los procesos cognitivos y mnemotécnicos que intervienen en la recuperación y apropiación de los contenidos.

Finalmente, los módulos cuentan con una presentación global del contenido el cual prepara al usuario para un aprendizaje integrado, es decir, un proceso en el que apropia los contenidos como un todo y establece conexiones entre las secuencias temáticas. Este se presenta como un mapa de navegación que flexibiliza y orienta los procesos de exploración, permitiendo así que el usuario tenga un mayor control sobre su itinerario de aprendizaje.

Por ser un tema novedoso y de alto impacto en la sociedad colombiana, este curso virtual tendrá un gran potencial y tiene como objetivo capacitar a más de dos mil usuarios finales por año. Una vez se encuentre habilitado en la plataforma Moodle de la Entidad, capacitará a docentes, personal administrativo de instituciones académicas y padres o tutores de niños y adolescentes en edad escolar, a través de la "Escuela de Padres SICEDUCA", programa que consiste en generar espacios académicos virtuales para padres que se encuentren en procesos de capacitaciones por parte de las instituciones académicas en las cuales se encuentran matriculados sus hijos.

\section{Desarrollo}

El curso se desarrolla partiendo de la elaboración de un plan estratégico bajo los estándares estipulados en el Project Management Institute (PMI), que gestiona la integración del proyecto con sus diferentes actores y en el que se verifica su alcance, basados en los tiempos, riesgos y requisitos establecidos para cada actividad. De esta forma, se conforman los equipos de trabajo, los comités operativos y los supervisores del proyecto quienes, según el plan táctico, establecen las reuniones, metas, objetivos, tiempos y entregables. Para tales efectos, se planearon tres fases. La fase I, Diseño; fase II, Desarrollo y Virtualización; y la fase III, Entrega y Cierre.

El proyecto se diseña teniendo una planeación transversal e iniciando con actividades primordiales como project charter, análisis de stakeholders, identificación de riesgos iniciales y plan de comunicaciones. En la ejecución del proyecto se hace un 
control de tiempos, recursos, presupuesto, según los hitos establecidos en el cronograma de trabajo, el cual está desarrollado en la herramienta Project.

Para tener un control y monitoreo efectivo, se desarrollan comités operativos con el equipo académico, el de desarrollo del curso, el equipo gerencial y los supervisores del proyecto. Allí se analizan y mitigan los riesgos existentes en el proyecto, se valida el avance y se generan alertas tempranas que permiten minimizar el impacto en caso de retrasos.

\section{Fase I - Diseño}

La fase de diseño tiene como insumo la ficha académica del curso, en la que los autores definen, según los lineamientos del diseñador instruccional, los aspectos fundamentales del curso. Posteriormente, se inicia el proceso de escritura en el que se hace un seguimiento pedagógico para que la redacción se conciba para un contexto virtual de aprendizaje. Este contenido se remite a la SIC para la aprobación por parte de los supervisores.

Entretanto, el equipo de Diseño Gráfico e Integración Multimedia junto con el diseñador instruccional desarrolla la propuesta de la interfaz gráfica y las herramientas de interactividad.

\section{Fase II - Desarrollo y Virtualización}

Dentro de esta fase se contempla la elaboración de un módulo introductorio que brinda al estudiante la posibilidad de familiarizarse con el curso, los objetos virtuales y la correcta navegación así como la información académica correspondiente a las generalidades del curso como lo son el objetivo general, introducción y mapa de navegación de los diferentes módulos con sus temas de estudio.

Cada contenido es verificado y validado por los supervisores del proyecto y el documento pasa por un proceso de corrección de estilo antes de seguir a la siguiente etapa de elaboración de guiones multimedia, donde se diseñan actividades interactivas, usando técnicas que facilitan el aprendizaje.

En la última etapa, el diseñador construye los guiones para los Objetos Virtuales de Aprendizaje. Después, el grupo de diseñadores gráficos e ingenieros dan vida a la propuesta plasmada en el guion, en los que se involucran actividades creativas e innovadoras que motivan al estudiante a finalizar el desarrollo del curso.

Los Objetos Virtuales de Aprendizaje (OVA) constituyen un paquete LMS (Learning Content Management System) que se exporta como SCORM, el cual permite capturar información del usuario en el proceso de aprendizaje (interacciones, contenido visualizado, actividades realizadas, puntaje de los juegos).

Dichos OVAs se diseñan, desarrollan e integran en la última versión disponible de Adobe Captivate 8.0.1.242 para Mac OS el cual permite exportar información para integración web (.swf y HTML5) y, además, generar un PDF interactivo que reproduce los contenidos generados en el OVA.

Las imágenes se crean en las últimas versiones disponibles de Adobe Creative Cloud; los videos se producen con Adobe After Effects CC 2015, Premiere Pro CC 2015 y se exportaron en los formatos correspondientes (.mov), se comprimen en Media Encoder CC 2015 en formatos optimizados para rápida reproducción y bajo tamaño en 


\section{CBIE-LACLO 2015}

Anais dos Workshops do IV Congresso Brasileiro de Informática na Educação (CBIE 2015)

almacenamiento (.mp4 codec: H264, .flv, .f4v). El material interactivo y el material que requiera redistribución en la interfaz se crea con programas especializados: Adobe After Effects CC 2015, Flash Professional CC and Mobile Device Packaging 2015, Adobe Edge Animate CC 2015.

\section{Fase III - Entrega y Cierre}

En esta fase se verifica que el aula virtual tenga los OVAs, cuestionarios, archivos descargables, material de apoyo y demás elementos requeridos para la ejecución del curso. Asimismo, se valida que esté activa la opción de la generación automática de certificados con los estándares requeridos por la SIC. Finalmente, se hace un entrenamiento a los funcionarios que la SIC designe en el manejo de los archivos fuente del curso y navegación de los mismos.

\section{Presentación del software}

El prototipo presentado consta de dos OVAs, correspondientes al módulo introductorio y al módulo temático 1. Los OVAs tienen una página de anclaje que contiene el menú principal:

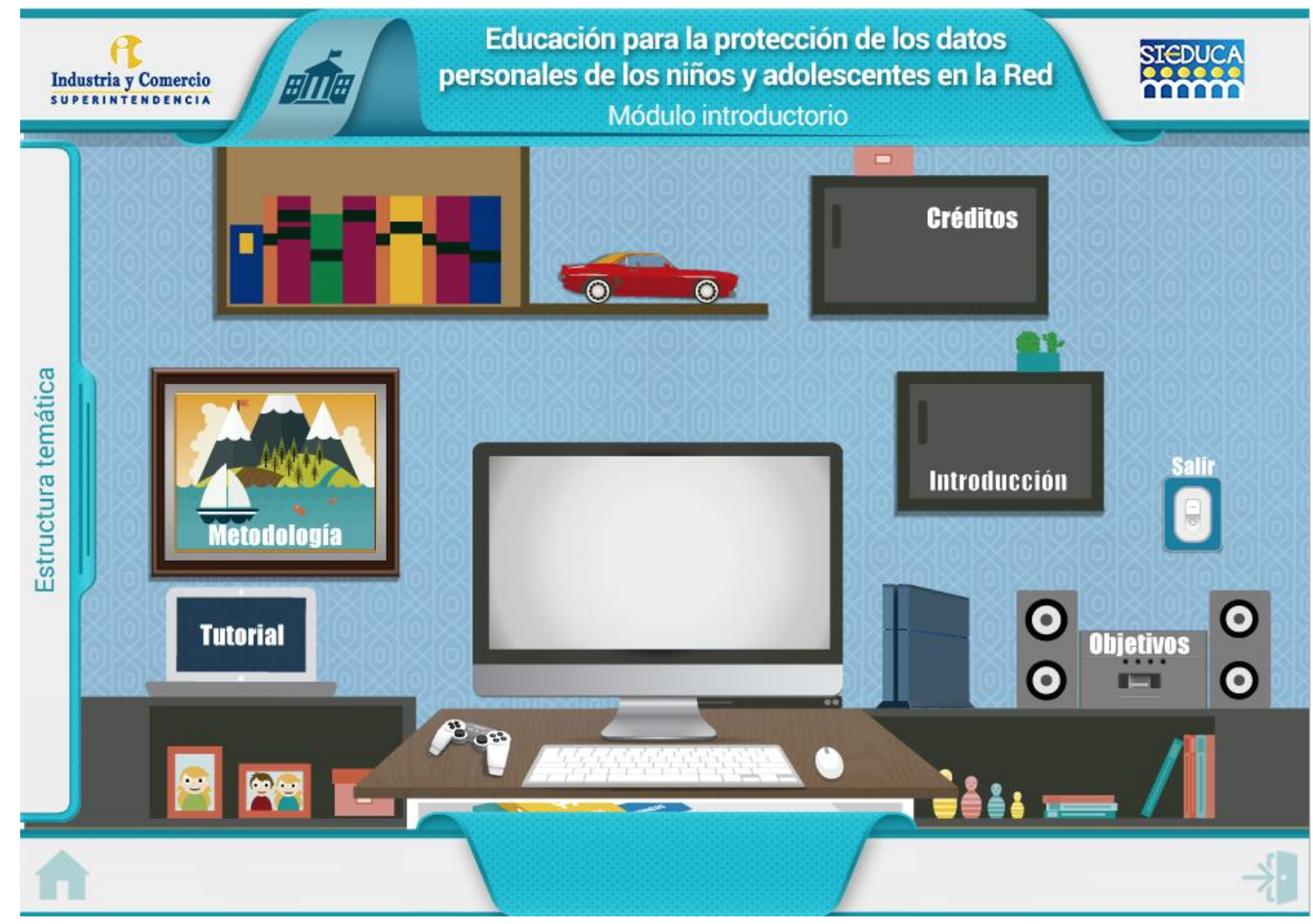

Figura 1. Menú principal módulo introductorio 
CBIE-LACLO 2015

Anais dos Workshops do IV Congresso Brasileiro de Informática na Educação (CBIE 2015)

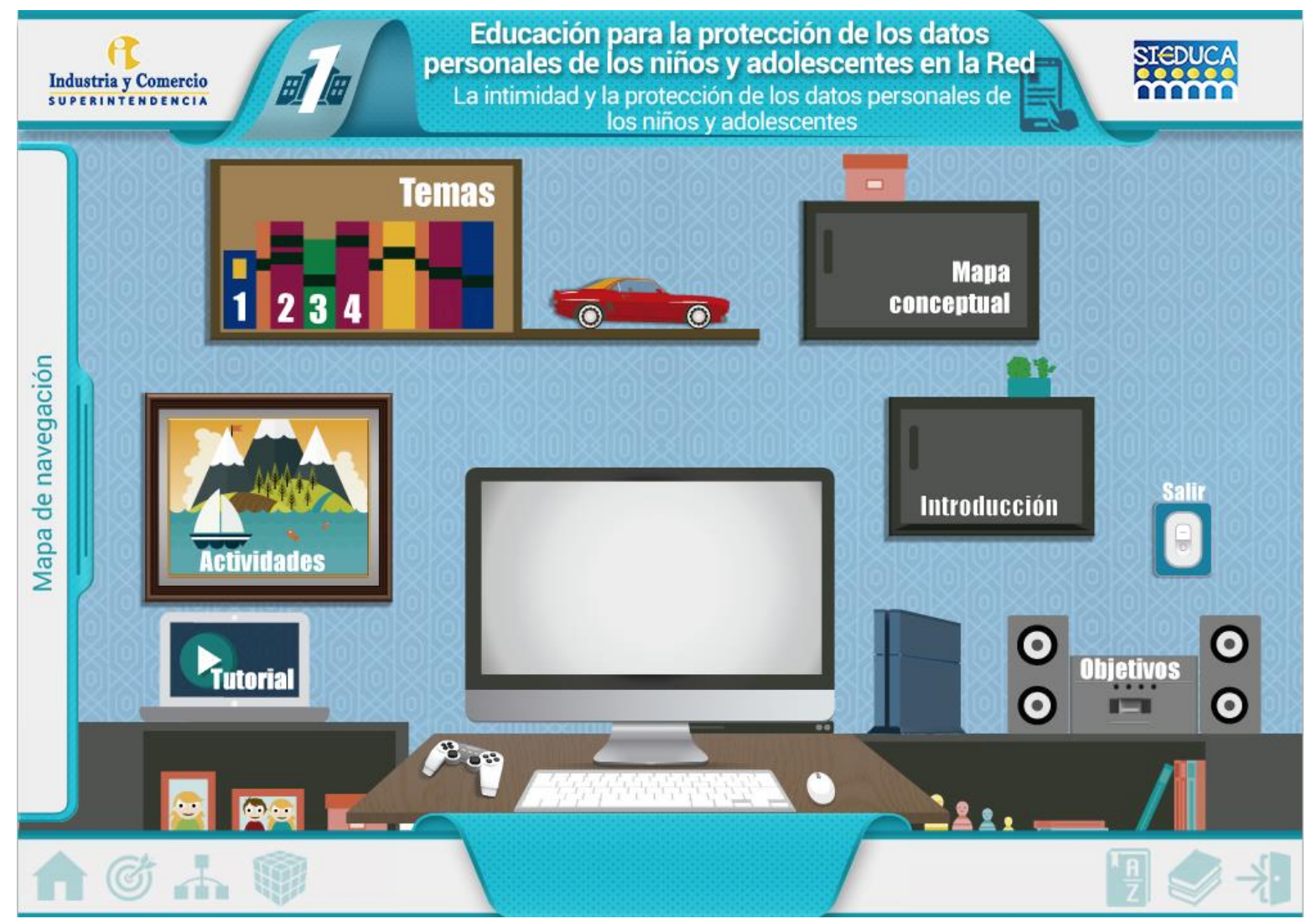

Figura 2. Menú principal módulo temático

En el módulo introductorio se exponen los aspectos fundamentales del curso. Esto le permite dilucidar la importancia del manejo de los datos personales en niños y adolescentes y la manera en que usted puede proteger a sus hijos o estudiantes en dicha materia.

El módulo uno se denomina: La intimidad y la protección de los datos personales de los niños y adolescentes. Con este OVA se empieza el estudio del curso ofreciendo a padres y docentes conceptos básicos asociados al manejo de datos personales en Colombia. En el siguiente video puede ver más detalladamente los elementos que encontrará en dicho módulo:

\section{https://www.dropbox.com/s/kwk1707j19u6idr/Video\%20Demostrativo..mp4?dl=0}

Adobe Captivate 8 genera contenidos y simulaciones virtuales con su licencia vitalicia, After Effects CC 2015 hace parte del paquete Creative Cloud 2015 de Adobe con licencia completa. Los contenidos que se muestran en el video demostrativo y en los módulos, hacen parte de la licencia de reconocimiento Creative Commons NoComercial.

\section{Consideraciones finales}

Para la Superintendencia de Industria y Comercio es fundamental velar por la seguridad de los datos personales y de la integridad de los niños y adolescentes de Colombia. En tal sentido, en su propósito de contribuir a la salvaguarda de los derechos de los consumidores, ha creado y puesto al servicio de la comunidad el curso Educación para la protección de los datos personales de los niños y adolescentes en la red, en una primera fase de virtualización de todos sus cursos presenciales. Este es el primer paso de un arduo 
CBIE-LACLO 2015

Anais dos Workshops do IV Congresso Brasileiro de Informática na Educação (CBIE 2015)

trabajo en el que la SIC se ha dado a la tarea de contribuir a las metas del Plan de Desarrollo: Todos por un nuevo país: Paz, Equidad y Educación, para hacer de nuestro país, un país más pacífico, próspero y educado.

\section{Bibliografía}

Asinten, J. (s.f.). Producción de contenidos para Educación Virtual. Argentina: Biblioteca Digital Virtual Educa. Recuperado el 4 de febrero del 2015, de: http://www.virtualeduca.org/documentos/manual del contenidista.pdf

Ministerio de Educación Nacional. (s.f.). Gestión de contenidos de educación virtual de calidad: Objetos de Aprendizaje. Recuperado el 4 de febrero del 2015, de: http://aprendeenlinea.udea.edu.co/lms/men/index.html

Plan Ceibal. (2009). Manual para el diseño y desarrollo de objetos de aprendizaje. Uruguay. Recuperado el 10 de febrero del 2015, de http://www.ceibal.edu.uy/UserFiles/P0001/ODEA/ORIGINAL/Exelearning.elp/GUI AObjetosCeibal09.pdf 